\title{
Application of HPLC for the Analysis of Secoisolariciresinol Diglucoside in Flaxseeds
}

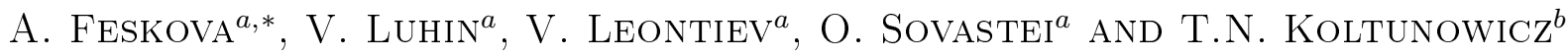 \\ ${ }^{a}$ Belarusian State University of Technology, Sverdlova 13a, 220050 Minsk, Republic of Belarus \\ ${ }^{b}$ Lublin University of Technology, Nadbystrzycka 38a, 20-618 Lublin, Poland
}

\begin{abstract}
High-pressure liquid chromatography - electrospray ionization-mass spectrometry coupled with the PDA detector was used for the analysis of secoisolariciresinol diglucoside in flaxseeds. The research was carried out using the spectrometer "Waters" on the BDS HYPERSIL column $\mathrm{C}_{18} 250 \times 4.6 \mathrm{~mm}$, with the diode-array PDA 996 and the mass-detectors Micromass ZQ 2000 with the electrospray ionization. As a mobile phase acetonitrile and water with $0.1 \%$ formic acid were used. The content of secoisolariciresinol diglucoside in the flaxseeds of 12 cultivar was determined.
\end{abstract}

DOI: $10.12693 /$ APhysPolA.123.834

PACS: $82.80 . \mathrm{Bg}, 82.80 . \mathrm{Qx}$

\section{Introduction}

Secoisolariciresinol diglucoside (SDG), or 2,3-bis (4hydroxy-3 methoxyphenyl) methyl]-1,4-butane diglucoside (Fig. 1) was identified in the flaxseeds in 1956 [1]. SDG is the main biologically active flaxseed lignan and one of the major precursors of mammalian lignans enterolactone and enterodiol, which plays an important role in protecting against hormone-dependent cancers (breast, prostate, thyroid etc.) as well as a number of other diseases (arteriosclerosis, osteoporosis, diabetes, etc.).

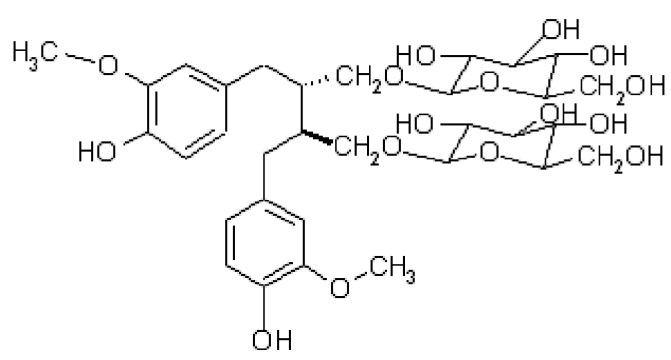

Fig. 1. The structure of the SDG.
Lignans as significant bioactive compounds with the potential application in pharmacy and nutrition demands appropriate analytical techniques for the extraction, separation and structural determination.

To determine the quantitative and qualitative composition of lignans in plant material, they must be separated from the plant matrix. Direct extraction and analysis are valid only for wood tissues where lignans are found in the free aglycone form. However, the analysis of other plants, which contain lignans, partly as a glycoside form (Fig. 2), is difficult since the glycoside bonds must be broken without degradation or alteration of lignan structures.

The destruction of plant matrix often requires monotonous operations, each of which carries a risk of contamination, sample loss and unexpected reactions of lignans. Nevertheless, the preparative and analytical methods were developed, an available equipment was set up and now it is quite possible to use safe methods of extraction and pre-treatment of plant samples. The general scheme applied for the lignan analysis of plant material is shown in Fig. 3.

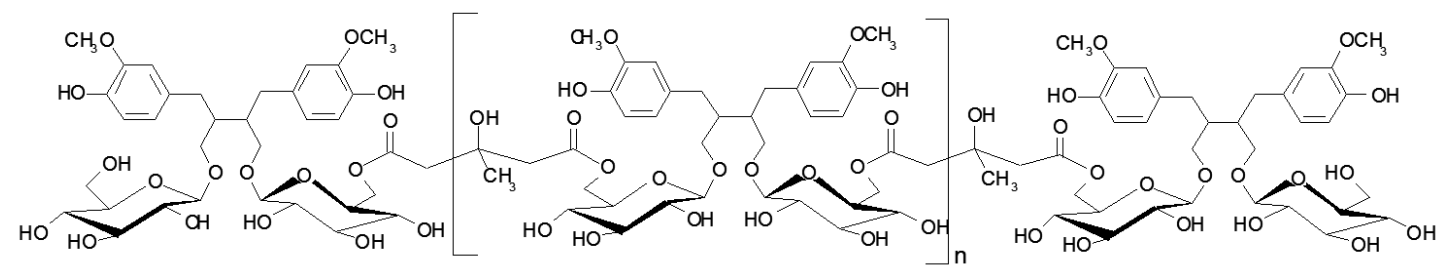

Fig. 2. The structure of the oligomer $(n=3 \div 5)$ SDG from flaxseeds. 


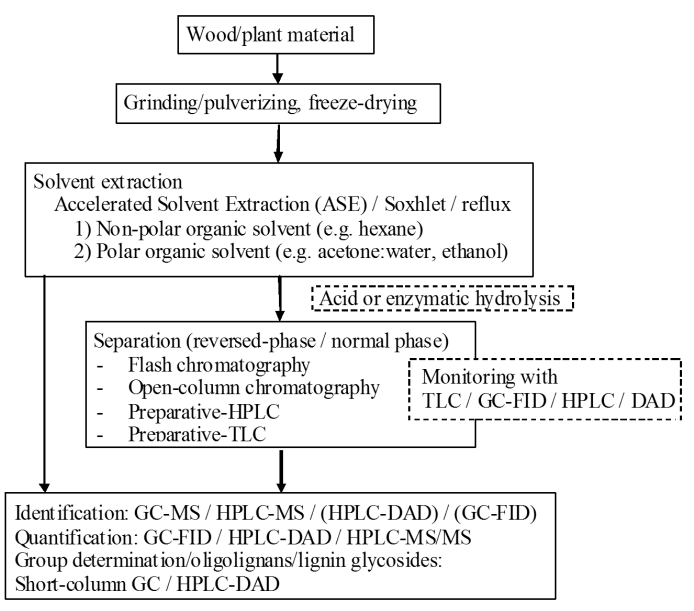

Fig. 3. General analytical scheme of the analysis and isolation of plant lignans.

matography (HPLC). HPLC is probably the most frequently used analytical technique for lignans, especially in the analysis of lignans in biological matrices. Most of the known lignans are medium polarity compounds, therefore for HPLC separation usually reversed-phase columns are used. The elution is carried out in the acidic mobile phase due to the acidity of phenolic groups of lignans. A mixture of methanol or acetonitrile and buffer solutions is used as a mobile phase.

For the detection of lignans the different analyzers are used. In the analysis of lignans in plant extracts UV detection can provide sufficient selectivity and sensitivity at the same wavelength. In more recent years UV detection is often used only in addition to other detection techniques, especially after the development of HPLC with mass spectrometry (MS), which mostly include also a UV diode-array detector (DAD) (or PDA detector) [2]. Mass spectrometry is a method of the research material by determining the ratio of the mass to charge $(\mathrm{m} / z)$ and the number of charged particles produced in a particular course of exposure [3]. MS analysis allows to carry out the preliminary identification of lignans and lignan glucosides [4]. In one of the earlier lignan HPLC-MS applications, HPLC-thermospray-MS coupled with or in combination with UV detection was used for lignan analysis. Nowadays the thermospray technique was replaced by the electrospray ionization (ESI) [2]. ESI is a technique used in mass spectrometry to produce ions. It is especially useful in producing ions from macromolecules because it overcomes the propensity of these molecules to fragment when ionized.

This paper deals with the analysis of content of SDG in flaxseeds of different cultivars using HPLC - ESI-MS coupled with the PDA detector.

\section{Experimental details and results}

The object of the investigation were the flaxseeds from the collection of the Institute of Genetics and Cytol- ogy National Academy of Science of the Republic of Belarus, which differed in seed production and have different ecological and geographical origins: Atalante (France); Blue Chip (Hungary); Omega (USA); McGregor, Somme, Raluca, Cyan (Poland); K-2398, K-6307 (China); Gold Flax (Canada), Vita, Solnechny (Republic of Belarus).

As generally known the main quantity of lignans is located in flaxseed hulls so the first step of the SDG isolation from flaxseeds was their destruction with the subsequent selection of flaxseed hulls fraction. The selected flaxseed hulls fraction was degreased with hexane in the Soxhlet apparatus. Then the step of neutralization was followed by the alkaline hydrolysis of the defatted fraction. Hydrolysis of the extracts is necessary so as most of SDG is the part of the polymeric glycosides. The extraneous substances were formed in carrying out an acid hydrolysis, complicating the chromatographic analysis [4]. Good results as for the isolation and analysis of enzymatic hydrolysis of SDG outcomes, high purity enzymes are required. Therefore, alkaline hydrolysis under mild conditions, which gives reproducible results was chosen [5]. The next step was the extraction with a mixture of ethyl:1.4-dioxane and evaporation. The obtained extracts were analyzed using the HPLC-MS spectrometer "Waters" on the BDS HYPERSIL column $\mathrm{C}_{18} 250 \times 4.6 \mathrm{~mm}$, with the diode-array PDA 996 and mass-detectors Micromass ZQ 2000 with ESI. The parameters of ionization were the following: capillary voltage $-3 \mathrm{kV}$, cone voltage $-40 \mathrm{~V}$, extractor voltage $5 \mathrm{~V}$; source temperature $-130^{\circ} \mathrm{C}$, desolvation temperature $-350^{\circ} \mathrm{C}$. The ESI/MS traces were recorded in positive and negative ions from $\mathrm{m} / z 100$ to 1000 . As the mobile phase there was used acetonitrile (A) and water with $0.1 \%$ formic acid (B) in the ratio $\mathrm{A}: \mathrm{A} 0-5 \mathrm{~min}-$ 30:70, 20-30 $\mathrm{min}-70: 30$ and 50-65 $\mathrm{min}-100: 0$. The elution rate was $1.0 \mathrm{ml} / \mathrm{min}$. The retention time of SDG is $14.92 \mathrm{~min}$ (Fig. 4).

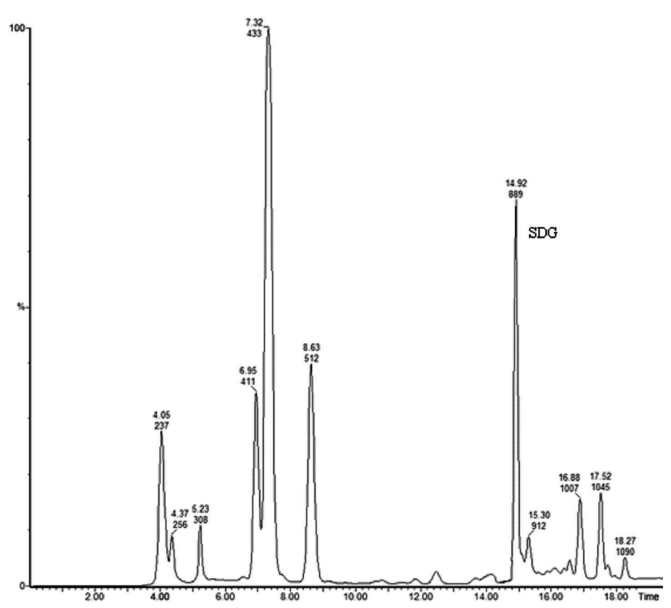

Fig. 4. The chromatogram of SDG.

The electronic spectrum of the component with the chromatographic peak retention time 14.92 min has the 
maximum at $280 \mathrm{~nm}$, which is typical of SDG [6] (Fig. 5). Due to the fact that the molecular weight of SDG is $686 \mathrm{Da}$, it was identified in the mass spectrum in the positive ions of the molecular ion $[\mathrm{M}+\mathrm{H}]^{+}$with $\mathrm{m} / z 687.95$ (Fig. 6a) and in the negative ions $[\mathrm{M}-\mathrm{H}]^{-}-$the ion with $m / z 685.92$ (Fig. 6b).

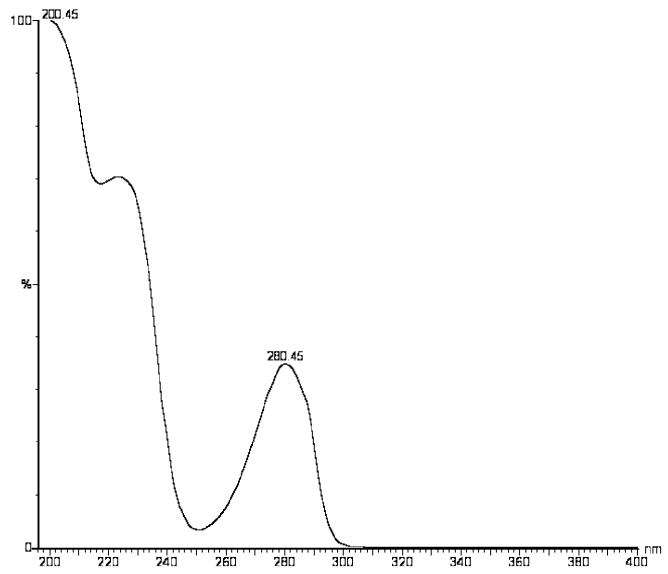

Fig. 5. The electronic spectrum of SDG.

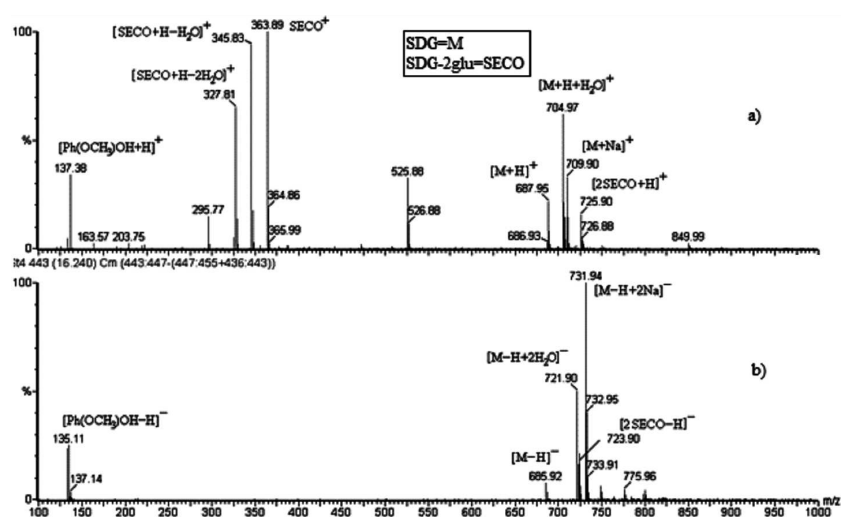

Fig. 6. Mass spectrum of SDG in the positive (a) and the negative (b) ions.

The peak with $m / z 363.89$ is the protonated aglycone of SDG $\left([\mathrm{M}+\mathrm{H}-2\right.$ glucose $\left.]=[\mathrm{SECO}]^{+}\right) \quad$ (secoisolariciresinol), the peak with $\mathrm{m} / z 327.81$ matches [SECO + $\left.\mathrm{H}-2 \mathrm{H}_{2} \mathrm{O}\right]^{+}$, the peak with $\mathrm{m} / z 345.83-[\mathrm{SECO}+\mathrm{H}-$ $\left.-\mathrm{H}_{2} \mathrm{O}\right]^{+}$(Fig. 6a). In the spectrum of negative ions (Fig. 6b) the signal with $m / z 721.90$ corresponds to the ion $\left[\mathrm{M}-\mathrm{H}+2 \mathrm{H}_{2} \mathrm{O}\right]^{-}$, the peak with $m / z 723.90$ is the ion $[2 \mathrm{SECO}-\mathrm{H}]^{-}$. The signals with $\mathrm{m} / \mathrm{z} 137.38$ and $\mathrm{m} / \mathrm{z}$ 135.11 in Figs. 6a and b, respectively, correspond to the hydroxy metoxybenzil ion $\left(\mathrm{Ph}\left(\mathrm{OCH}_{3}\right) \mathrm{OH}\right)$. For the quantitative determination of SDG the calibration curve constructed from the standard solutions of commercial preparation of SDG (ChromaDex) was used.

TABLE

The content of SDG in the flaxseeds of different cultivars.

\begin{tabular}{c|c}
\hline \hline Name of cultivar & $\begin{array}{c}\text { The content of SDG in the } \\
\text { defatted fraction }[\mathrm{mg} / \mathrm{g}]\end{array}$ \\
\hline K 2398 & $8.440 \pm 0.2992$ \\
Atalante & $11.097 \pm 0.8037$ \\
Blue Chip & $6.996 \pm 0.3880$ \\
Cyan & $9.964 \pm 0.5202$ \\
Gold Flax & $14.414 \pm 0.8204$ \\
McGregor & $10.199 \pm 0.6664$ \\
Omega & $9.177 \pm 0.1729$ \\
Raluca & $12.241 \pm 1.2984$ \\
Somme & $8.978 \pm 0.5107$ \\
Vita & $8.6801 \pm 0.7255$ \\
K-6307 & $5.3925 \pm 0.1447$ \\
Solnechny & $12.290 \pm 0.4260$
\end{tabular}

The results of quantitative research on the content of SDG in flaxseeds of different cultivars are presented in Table. They show the highest content of SDG in flaxseed of Gold Flax, Raluca and Solnechny cultivars.

\section{Conclusion}

The obtained results can be used for the quantitative and qualitative analyses of lignans in plant facilities and herbal medicine as well as in the process of preparation of new generation drugs with the high anti-oxidant, anti-allergic and anti-tumor properties.

\section{References}

[1] J.E. Bakke, H.J. Klosterman, Proc. North Dakota Acad. Sci. 10, 18 (1956).

[2] S.M. Willför, A.I. Smeds, B.R. Holmbom, J. Chromatogr. A 1112, 64 (2006).

[3] W. Henderson, J.S. Mc Indoe, Mass Spectrometry of Inorganic and Organometallic Compounds, Wiley, Chichester, 2005 p. 271.

[4] T. Silica, H.B. Niemeyer, D.M. Honig, M. Metzler, J. Agric. Food Chem. 51, 1181 (2003).

[5] P. Johnsson, A. Kamal-Eldin, L.N. Lundgren, P. Aman, J. Agric. Food Chem. 48, 5216 (2000).

[6] B. Madhusudhan, D. Wiesenborn, J. Schwarz, K. Tostenson, J. Gillepse, Lebensmittel- Wissenschaft-und-Technologie 33, 268 (2000). 